\title{
Recurrent Strokes as the First Presentation of Occult Pancreatic Cancer; Trousseau Syndrome: A Case Report
}

\author{
Firas Al Nidawi ${ }^{a} \quad$ Mohamed Wael Mohamed $^{a} \quad$ Fatima Taha $^{a}$ \\ Daher Alaraba Amr Elsayed M. Hussein ${ }^{b}$ \\ aInternal Medicine Department, King Hamad University Hospital, Muharraq, Kingdom of \\ Bahrain; ${ }^{b}$ Radiology Department, King Hamad University Hospital, Muharraq, Kingdom of \\ Bahrain
}

\section{Keywords}

Trousseau syndrome $\cdot$ Pancreatic cancer $\cdot$ Recurrent strokes

\begin{abstract}
Cerebrovascular disorders (CVDs) are widespread in cancer patients, exacerbating their condition and lowering their prognosis. Approximately $15 \%$ of cancer patients have a coexisting CVD. Stroke may occur after the initial cancer diagnosis or before the diagnosis of malignant disease. The underlying causes of stroke in cancer patients are distinct from those in noncancer patients and are related to both cancer itself and the type of treatment. Cardio-embolism, large vessel atherosclerosis, and small vessel occlusion have all been identified as important causes of ischemic stroke, but nonbacterial thrombotic endocarditis has been documented only infrequently. We present a case of a 64-year-old female with recurrent ischemic stroke of unknown cause and involving different arterial territories, who was discovered to have metastatic pancreatic cancer. The initial workup with brain imaging revealed multiple acute/ subacute ischemic strokes and a negative cardiac assessment. Accordingly, she underwent a whole-body positron emission tomography-computed tomography which revealed a hypermetabolic mass lesion in the pancreaticoduodenal area. Our case indicates that cancer-associated stroke should be seriously evaluated. It demonstrates the critical nature of contemplating hidden cancer in survivors of ischemic stroke and identifies factors that may necessitate further investigation as part of a comprehensive approach to ischemic stroke, especially embolic stroke of unknown sources.
\end{abstract}


Al Nidawi et al.: Occult Pancreatic Cancer Diagnosed Following Acute Ischemic Stroke

\section{Introduction}

Systemic cancer and ischemic stroke are both common diseases that are among the leading causes of death in the elderly [1]. The world's continually growing geriatric population is expected to result in a rise in new cancer cases. Additionally, advancements in treatment practice (cancer medicine) have the potential to increase survival, and the number of cancer patients is predicted to increase [1].

Among ischemic stroke patients, $10 \%$ had known cancer and $3 \%$ had hidden malignancy. In the USA, nationwide inpatient data revealed that approximately 1 in every 10 hospitalized ischemic stroke patients has comorbid cancer, and there was a significant decrease in ischemic stroke hospitalization in the cancer-negative group, but a steady increase in ischemic stroke hospitalization with a cancer diagnosis [2]. Hidden cancer was diagnosed during follow-up after acute ischemic stroke in $2.8 \%$ (20.4\% in embolic stroke of unknown sources [ESUS]) of Korean patients [1, 3], 2.1\% (5.3\% in ESUS) of Spanish patients [1, 4], 4.3\% of Norwegian patients $[1,5]$, and $3.0 \%$ of Japanese patients $[1,6]$, indicating that hidden malignancy may be a cause of stroke.

Trousseau was the first to report venous thrombosis, which occurred during his own pancreatic cancer treatment. His name was used to define any thromboembolic episode as the first sign of hidden malignancy [7]. Cancer-related consequences such as coagulation abnormalities and infections, as well as therapeutic and diagnostic procedures can all produce strokes [8].

There are no evidence-based guidelines for treatment of cancer-related stroke. Anticoagulation therapy, like in venous thromboembolism, is essential in individuals with active malignancy and ischemic stroke [9]. Effective hypercoagulability correction played a protective influence in the survival of cancer-related stroke patients [9]. This case report emphasizes the importance of considering underlying malignancy in cases of ESUS.

\section{Case Presentation}

A 62-year-old Bahraini woman presented to our hospital $6 \mathrm{~h}$ after experiencing weakness and numbness in her right upper limb that recovered spontaneously. Her vital signs were normal apart from elevated blood pressure of 170/85 mm Hg. The neurological examination was unremarkable. Accordingly, magnetic resonance imaging (MRI) and magnetic resonance angiography brain had been performed and they showed multiple small foci of restricted diffusion on cortical, subcortical regions of frontal, parietal, and occipital hemispheres, and both cerebellar hemispheres, with normal magnetic resonance angiography (Fig. 1). Cardiovascular workup, including transthoracic echocardiography, transoesophageal echocardiography (TEE), and a 24-h Holter monitor were all normal. Initially dual antiplatelets (aspirin and clopidogrel) were commenced alongside statin and antihypertensive medications. However, after reviewing the MRI and given the possible embolic nature, a decision was made to switch the patient from antiplatelets to rivaroxaban $20 \mathrm{mg}$ once daily which she was discharged on.

Two weeks later, she presented with a transient worsening in her right upper limb associated with incoherent speech lasted around $1 \mathrm{~h}$. The new MRI-diffusion-weighted imaging did not show any new stroke. Her laboratory investigations including protein C, protein S, anti-nuclear antibodies, and erythrocyte sedimentation rate were all normal. A 72-h Holter test revealed a sinus rhythm. Decision was made to continue rivaroxaban $20 \mathrm{mg}$ once daily and to add aspirin as well.

One week later, she presented to the Emergency Department with newly developed right sided weakness and incomprehensible speech that began approximately $4 \mathrm{~h}$ prior to her presentation. Clinical examination showed clinical characteristics consistent with large vessel occlusion with National Institutes of Health Stroke Scale (NIHSS) score of 7. Computed tomography

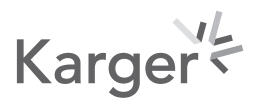




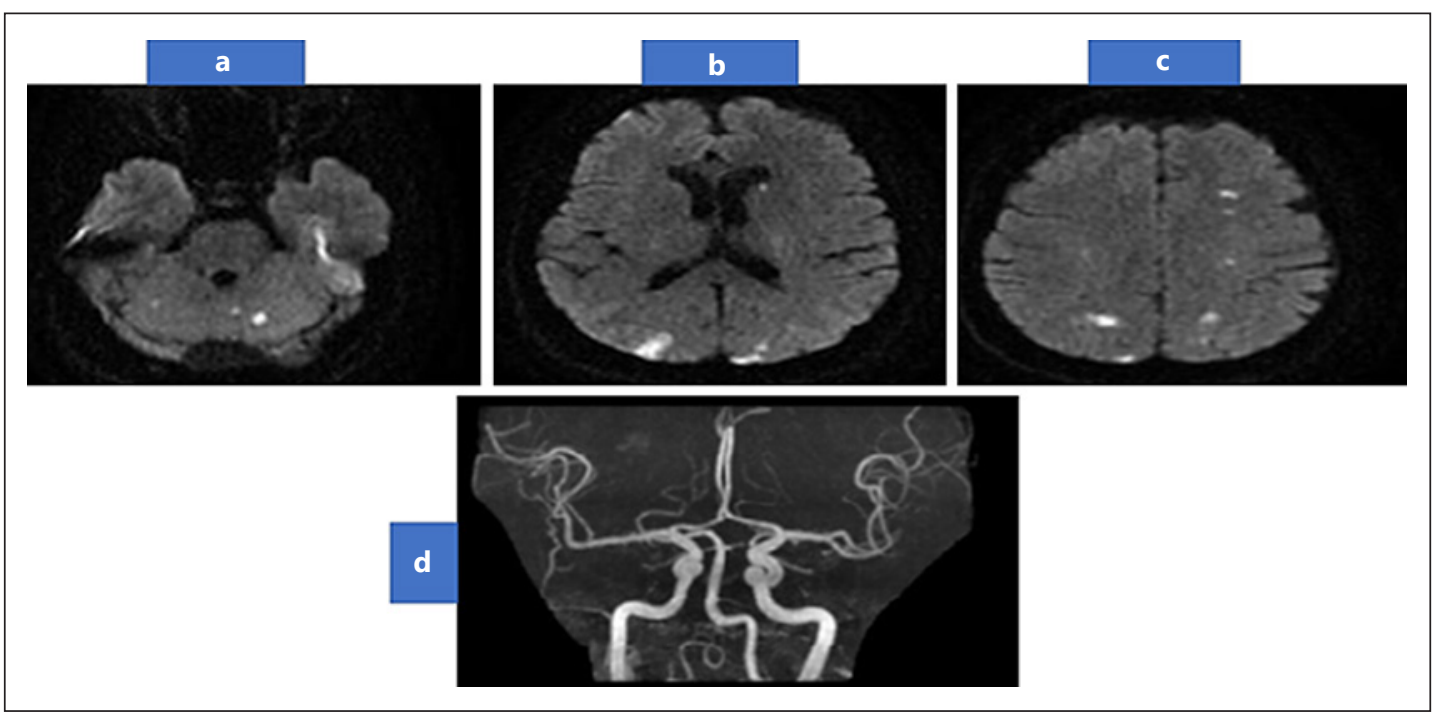

Fig. 1. DWI-MRI brain showing multiple small foci of restricted diffusion on cortical, subcortical regions of frontal, parietal, and occipital hemispheres (a-b). In addition to both cerebellar hemisphere (c), with normal MRA (d). DWI, diffusion-weighted imaging; MRI, magnetic resonance imaging; MRA, magnetic resonance angiography.

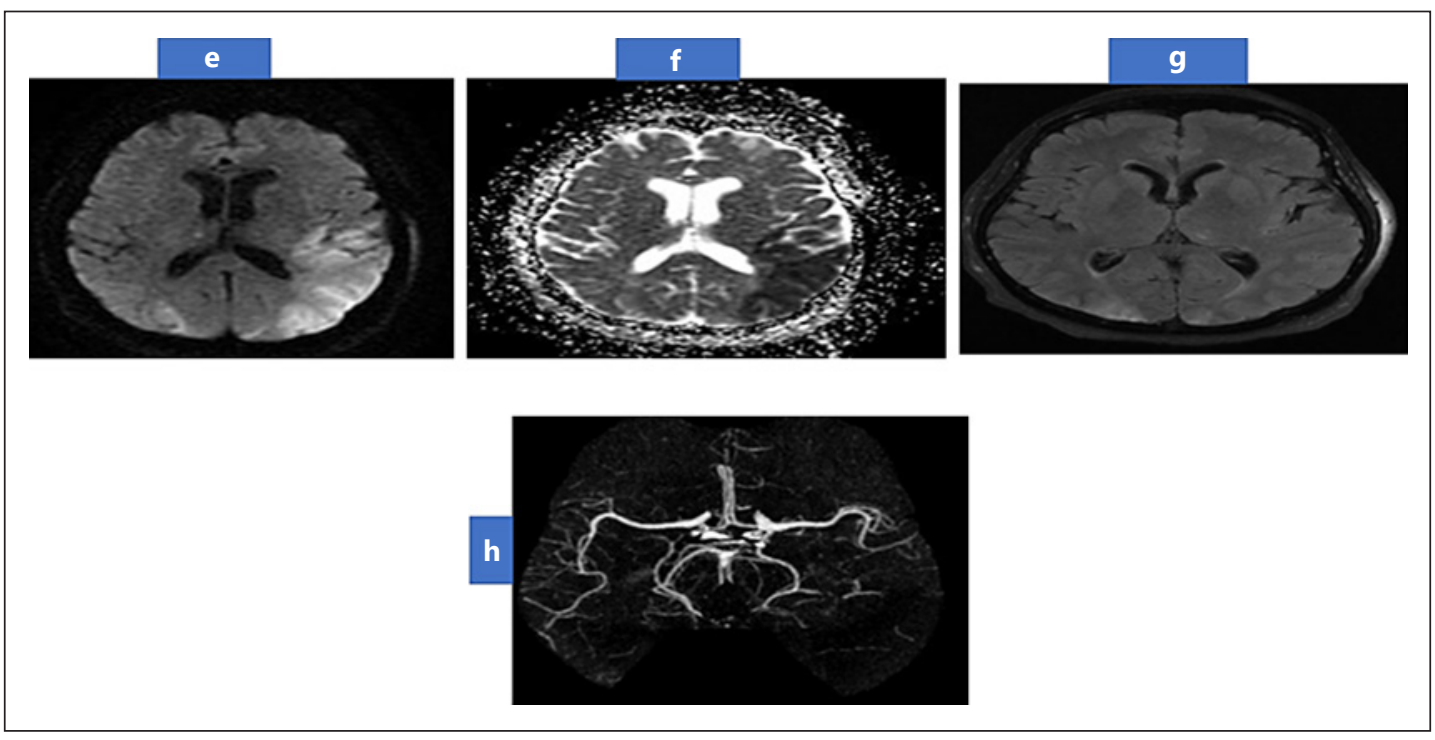

Fig. 2. MRI Brain showing restricted diffusion in left parieto-occipital region in DWI and ADC (e, f), with normal FLAIR (g) suggestive of acute ischemic. MRA (h) showing occluded distal M3 of left middle cerebral artery with attenuated distal branches. MRI, magnetic resonance imaging; MRA, magnetic resonance angiography; DWI, diffusion-weighted imaging.

(CT) angiography showed occlusion of the left middle cerebral artery's inferior branch (Fig. 2). Thrombectomy was then undertaken, but conventional angiography showed spontaneous recanalization of the occluded artery. Acute parieto-occipital infarction was discovered on MRI brain. Following admission and as part of the workup for recurrent strokes of unexplained origin, a positron emission tomography CT scan of the body (Fig. 3) was performed to rule out any underlying malignancy. The scan revealed a hypermetabolic mass lesion (neoplasm) at the 


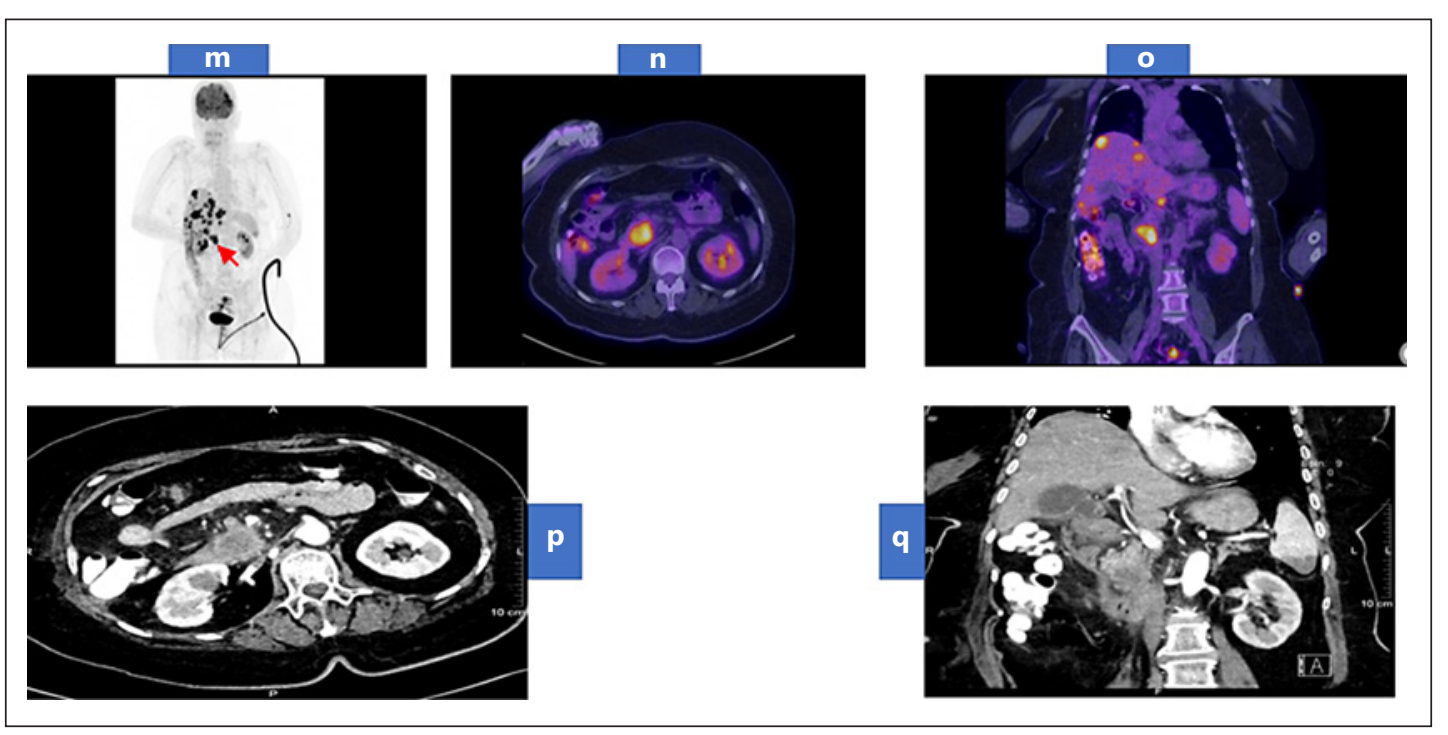

Fig. 3. Whole-body maximum intensity projection of FDG PET scan ( $\mathbf{m}$ ) showing a hypermetabolic primary neoplasm at the pancreatic head (arrow) associated with multiple variable-sized hypermetabolic hepatic focal deposits and less avid lesion at the lower pole of the spleen. Trans-axial fused FDG PET/CT images of the abdomen (n) showing the hypermetabolic primary pancreatic head mass. Coronal fused FDG PET/CT images of the abdomen and pelvis (o) showing the hypermetabolic primary pancreatic head neoplasm associated with multiple hypermetabolic hepatic deposits. Contrast-enhanced CT of the abdomen arterial phase (p) axial view showing a large hypodense and hypovascular mass lesion arising from the pancreatic uncinated process. (q) coronal view showing multiple hypodense hepatic focal deposits and hypodense splenic focal lesion at its lower pole. PET, positron emission tomography; CT, computed tomography.

pancreatic head associated with multiple variable-sized hypermetabolic hepatic focal deposits and less avid lesion at the lower pole of the spleen (Fig. 3). Following that, a CT abdomen was performed and showed a hypodense mass lesion in the pancreas uncinate process that was most likely malignant, as well as several metastatic liver lesions (Fig. 3).

The patient intended to undergo further treatments to achieve a tissue diagnosis, but 9 days after admission, she developed sudden onset left-sided hemiplegia. CT angiography showed M1 occlusion of the right middle cerebral artery (Fig. 4); as a result, a thrombectomy with a modified thrombolysis in cerebral infarction scale (m-TICI) score of $2 \mathrm{~A}$ was performed. A new TEE showed an echogenic shadow of approximately $2.1 \mathrm{~cm}^{2}$ in the right ventricular cavity, consistent with a right ventricular clot, but repeated blood cultures were negative. The patient did not meet the diagnostic criteria for infective endocarditis. As a result, the diagnosis of nonbacterial thrombotic endocarditis was made in association with thromboembolic phenomena caused by an underlying malignancy. Initially the anticoagulation was kept on hold. Subsequently, unfractionated heparin infusion was initiated. She developed heparininduced thrombocytopenia and was hence switched to argatroban in addition to aspirin. Unfortunately, the patient's condition deteriorated further, she suffered several complications, including pulmonary embolism, and died 2 months after admission.

\section{Discussion}

Trousseau is credited with being the first to describe cancer-related thrombosis in his 1865 report on migrating thrombophlebitis in the context of visceral malignancy [10]. 


\section{Case Reports in Oncology}

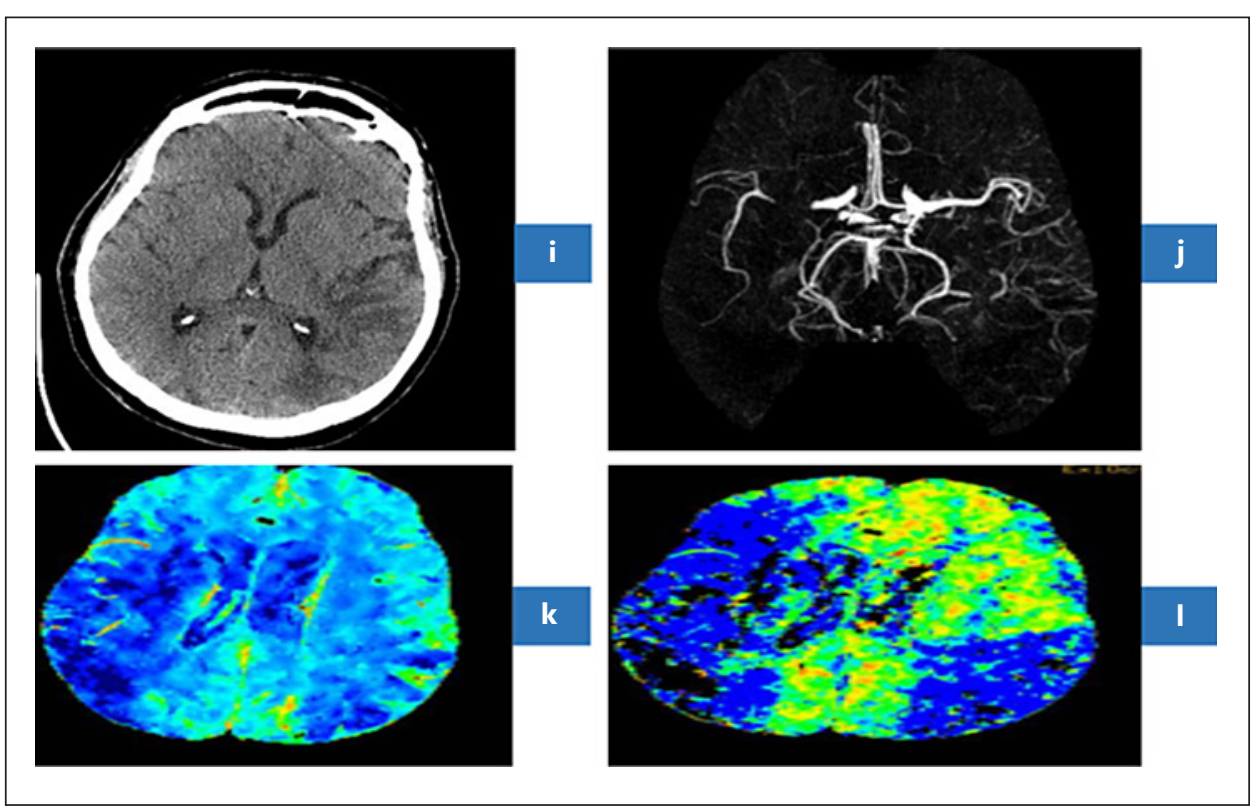

Fig. 4. Axial NCT Brain (i) revealed subacute stroke in the territory of inferior branch of left MCA. CT angiography (j) revealed M1 occlusion of right MCA. CT perfusion revealed decrease cerebral blood flow (k) and decreased mean transient time (I) in the territory of right MCA indicating acute right MCA ischemic stroke. CT, computed tomography; MCA, middle cerebral artery.

Cancer can lead to stroke by several major pathophysiological mechanisms which can be directly related to the cancer or by the therapeutic and diagnostic interventions including chemotherapy, radiotherapy, invasive procedures, and supportive therapies like hematopoietic stem cell transplantation [8]. Hypercoagulability and inflammation associated with cancer may induce acute ischemic stroke by a variety of mechanisms, including paroxysmal embolism, nonbacterial thrombotic endocarditis, and intravascular coagulation [11]. Adenocarcinomas may also secrete mucin into the bloodstream, which lead to the production of platelet-rich microthrombi by interacting with cell adhesion molecules [11]. Furthermore, cancer might cause stroke by direct tumor effects like arterial and venous invasion, sinvenous thrombosis, leptomeningeal metastasis, tumor emboli, blood vessel compression, tumor bed edema, etc. [8].

When acute ischemic strokes occur in the presence of occult cancer, they tend to recur more frequently and have a worse prognosis than strokes that do not occur in the presence of cancer [12]. Around 1 in every 5 ischemic infarcts in patients with diffusion-weighted imaging lesions involving 3 vascular regions are caused by malignancy. In the absence of an identifiable embolic source, $75 \%$ of cases involve ischemia infarction linked with cancerrelated hypercoagulation [13].

The management of acute ischemic stroke in cancer and noncancer patients has not been proven to differ significantly; cancer should not be considered an absolute contraindication to intravenous thrombolysis [8]. In terms of prevention, the clinician should evaluate the benefits and drawbacks of an anticoagulant therapy plan before implementing it. So far, low molecular weight heparin has been the preferred option [14].

Our patient's medical history revealed no evidence of malignancy. We investigated extensively to identify an etiology for her many ischemia lesions at different ages, involving both cerebellar hemispheres, in addition to the cerebellum on her brain MRI. The normal arterial brain images and routine stroke workup including transthoracic echocardiography and TEE, 
prompted us to investigate for rare causes of stroke which include occult malignancy. Wholebody positron emission tomography followed by CT abdomen confirmed the presence of pancreatic cancer. Most pancreatic malignancies are invasive ductal carcinomas that infiltrate adjacent organs [15]. It is rarely diagnosed in its early stages, and as a result, it is mostly incurable in the majority of instances [15]. The initial step in treating Trousseau's condition is to remove the underlying tumor, although this is not always feasible because the tumor is frequently unresectable [15]. Despite all preventive measures, including low molecular weight heparin and novel oral anticoagulants, the patient experienced repeated strokes followed by pulmonary embolism and died 2 months after admission.

In conclusion, multiple cerebral infarctions may be the earliest signs of an occult malignancy that has gone untreated. If there are multiple infarctions in both the anterior and posterior cerebral circulations or in the bi-hemispheric circulations, hypercoagulability due to an undiscovered malignancy should be addressed in patients with an unknown cause.

\section{Conclusion}

Multiple cerebral infarctions may be the earliest signs of an occult malignancy. Our case demonstrates how recurrent stroke can be the first clinical manifestation of a metastatic tumor, especially in the absence of conventional vascular risk factors, as well as the importance and implications of diagnosing malignancy-related strokes, and the practical difficulties clinicians face in managing an increasingly common condition with no clear guidelines.

\section{Statement of Ethics}

Written informed consent from the next of kin was obtained to publish this case and accompanying images. Ethical approval as obtained by the IRB research committee at King Hamad University Hospital (reference No. 19-294) to publish this case.

\section{Conflict of Interest Statement}

The authors declare that there is no conflict of interest.

\section{Funding Sources}

The authors declare that no funding was received for this case report.

\section{Author Contribution}

Firas Alnidawi contributed to planning the outline of the case report, critical revision, editing, and final approval. Mohamed Wael Mohamed contributed to drafting the case report and literature review. Fatima Taha contributed to drafting the case report and literature review. Daher Alarab contributed to literature review and critical revision. Amr Elsayed contributed to providing the images, commenting on the findings, and critical revision.

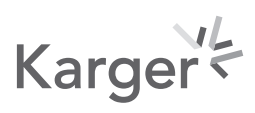




\section{Data Availability Statement}

All data that were used in this case report is available upon request.

\section{References}

1 Bang OY, Chung JW, Lee MJ, Seo WK, Kim GM, Ahn MJ, et al. Cancer-related stroke: an emerging subtype of ischemic stroke with unique pathomechanisms. J Stroke. 2020;22(1):1-10.

2 Sanossian N, Djabiras C, Mack WJ, Ovbiagele B. Trends in cancer diagnoses among inpatients hospitalized with stroke. J Stroke Cerebrovasc Dis. 2013;22:1146-50.

3 Selvik HA, Thomassen L, Logallo N, Næss H. Prior cancer in patients with ischemic stroke: the Bergen NORSTROKE study. J Stroke Cerebrovasc Dis. 2014;23:919-25.

4 Kim SJ, Park JH, Lee MJ, Park YG, Ahn MJ, Bang OY. Clues to occult cancer in patients with ischemic stroke. PLoS One. 2012;7:e44959.

5 Cocho D, Gendre J, Boltes A, Espinosa J, Ricciardi AC, Pons J, et al. Predictors of occult cancer in acute ischemic stroke patients. J Stroke Cerebrovasc Dis. 2015;24:1324-8.

6 Selvik HA, Thomassen L, Bjerkreim AT, Næss H. Cancer-associated stroke: the Bergen NORSTROKE study. Cerebrovasc Dis Extra. 2015;5:107-13.

7 Ansari D, Ansari D, Andersson R, Andrén-Sandberg Å. Pancreatic cancer and thromboembolic disease, 150 years after Trousseau. Hepatobil Surg Nutr. 2015;4:325-35.

8 Dardiotis E, Aloizou AM, Markoula S, Siokas V, Tsarouhas K, Tzanakakis G, et al. Cancer-associated stroke: pathophysiology, detection and management (Review). Int J Oncol. 2019;54(3):779-96.

9 Lee MJ, Chung JW, Ahn MJ, Kim S, Seok JM, Jang HM, et al. Hypercoagulability and mortality of patients with stroke and active cancer: the OASIS-CANCER Study. J Stroke. 2017;19:77-87.

10 Varki A. Trousseau's syndrome: multiple definitions and multiple mechanisms. Blood. 2007;110(6):1723-9.

11 Dearborn JL, Urrutia VC, Zeiler SR. Stroke and cancer: a complicated relationship. J Neurol Transl Neurosci. 2014;2:1039.

12 Navi BB, Iadecola C. Ischemic stroke in cancer patients: a review of an underappreciated pathology. Ann Neurol. 2018;83:873-83.

13 Finelli PF, Nouh A. Three-territory DWI acute infarcts: diagnostic value in cancer- associated hypercoagulation stroke (Trousseau Syndrome). AJNR Am J Neuroradiol. 2017;37:2033-6.

14 Van Es N, Büller HR. Using direct oral anticoagulants (DOACs) in cancer and other high risk populations. Hematology Am Soc Hematol Educ Program. 2015;2015:125-31.

15 Razmeh S, Ghurchian Z, Almasi M, Habibi AH, Rohani M. Rare presentation of pancreatic cancer: a case report. Int J Cancer Manag. 2017 Nov;10(11):e9052. 